\title{
Análise dos instrumentos de gestão elaborados pelas Secretarias Municipais de Saúde de Santa Catarina no período de 2014 a 2017
}

\author{
Analysis of management tools elaborated by the Municipal Health \\ Departments of Santa Catarina from 2014 to 2017
}

Cássio Noboro Fuginami', Claudia Flemming Colussi², Angela Maria Blatt Ortiga ${ }^{\mathbf{3}}$

DOI: 10.1590/0103-1104202012620

\begin{abstract}
RESUMO Os instrumentos de gestão foram instituídos no Sistema Único de Saúde como ferramentas norteadoras da gestão, assegurando a participação social na sua elaboração e apreciação. O Plano de Saúde, a Programação Anual de Saúde (PAS) e o Relatório Anual de Gestão (RAG) devem ser elaborados conforme estrutura e prazos definidos pelo Sistema de Planejamento do SUS. Considerando sua importância, o objetivo deste estudo foi analisar a estrutura mínima dos instrumentos de gestão elaborados pelas Secretarias Municipais de Saúde do estado de Santa Catarina. Trata-se de uma pesquisa exploratória, quantitativa, realizada por meio de análise documental. Foram analisados o Plano Municipal de Saúde de 2014 a 2017; a PAS e o RAG referentes ao ano de 2017, anexados ao Sistema de Apoio à Construção do Relatório de Gestão. No total, 288 municípios possuíam o PMS, e, destes, 136 a estrutura mínima. Quanto ao RAG, 248 municípios apresentaram o instrumento, dos quais, 125 possuíam a PAS. Identificou-se que a maior parte dos municípios não construiu adequadamente os instrumentos de gestão ou não os enviou nos prazos estabelecidos. A falta de resoluções de aprovação dos instrumentos por parte dos Conselhos de Saúde denota a fragilidade da participação social na construção e no acompanhamento da execução desses instrumentos.
\end{abstract}

PALAVRAS-CHAVE Planejamento em saúde. Gestão em saúde. Diretrizes para o planejamento em saúde. Planejamento estratégico.

1 Secretaria Municipal da Saúde de Luiz Alves - Luiz Alves (SC), Brasil. cassiofuginami@hotmail. com

2 Universidade Federal de Santa Catarina (UFSC) -

Florianópolis (SC), Brasil.

3 Universidade do Estado de Santa Catarina (Udesc)

- Ibirama (SC), Brasil.
ABSTRACT The management tools were established in the Unified Health System as guiding management tools, ensuring social participation in its elaboration and appreciation. The Health Plan, the Annual Health Schedule (AHS) and the Annual Health Report (AHR) must be prepared according to the structure and deadlines defined in the SUS Planning System. Considering its importance, the objective of this study was to analyze the minimum structure of the management tools elaborated by the Municipal Health Departments of the state of Santa Catarina. This is an exploratory, quantitative research, carried out through document analysis. The Municipal Health Plan from 2014 to 2017; the AHS and the AHR, relating to the year 2017 were analyzed, attached to the Management Report Construction Support System. In total, 288 counties had the MHP and, of these, 136 had the minimum structure. Regarding the AHR, 248 cities presented the tool, of which 125 had the AHS. It was identified that most cities did not properly build management tools or sent them within the set deadlines. The lack of resolutions approving the instruments by the Health Councils indicates the fragility of social participation in the construction and monitoring of the implementation of these tools. 


\section{Introdução}

O planejamento no Sistema Único de Saúde (SUS) é requisito legal, que expressa as responsabilidades dos gestores das três esferas do governo com relação à saúde da população. As Leis no 8.080 e no 8.142, de 1990, estabelecem que o planejamento deve ser ascendente e articulado, obedecendo as ações programáticas e a previsão orçamentária do Plano de Saúde ${ }^{\mathbf{1}, 2}$.

Visando ao fortalecimento do planejamento no SUS, o Ministério da Saúde (MS) instituiu, em 2006, o Pacto pela Saúde ${ }^{3}$, que, através do Pacto de Gestão, implementou o Sistema de Planejamento do SUS (PlanejaSUS) ${ }^{4}$, que tem por finalidade coordenar o processo de planejamento de forma contínua, participativa, integrada e articulada entre os três entes federativos.

O PlanejaSUS foi um marco para a consolidação do planejamento no SUS, atribuindo aos gestores locais mais responsabilidades e potencializando os instrumentos de gestão (Plano de Saúde, Programação Anual de Saúde, Relatório Anual de Gestão) como norteadores e condutores no processo de tomada de decisões e aperfeiçoamento na efetividade das ações e na avaliação dos serviços prestados ${ }^{\mathbf{5}, \mathbf{6}}$.

Com a perspectiva de aperfeiçoar o processo de planejamento, o MS atualizou a Portaria MS/GM n ${ }^{\circ} 399 / 06$ e criou a Portaria MS/GM $\mathrm{n}^{\mathrm{o}} 2.135 / 13$, que estabelece diretrizes para o planejamento do SUS. Essa portaria reafirma o planejamento como ferramenta de construção ascendente, com incentivo à participação da comunidade, compatível com os instrumentos de governo e destacando a utilização dos instrumentos de gestão do SUS7,8.

O Plano de Saúde (PS) é o instrumento central de planejamento do gestor, elaborado a partir das necessidades de saúde da população, considerando, entre outras, as diretrizes propostas pelos conselhos e conferências de saúde. Possui importante papel de monitoramento e na avaliação das ações, tendo como base o planejamento estratégico-situacional9,10.
A Programação Anual de Saúde (PAS) operacionaliza as intenções expressas do PS, anualizando suas metas e prevendo a alocação de recursos orçamentários. Desse modo, a PAS apresenta importante compatibilidade com as Leis de Diretrizes Orçamentárias (LDO) do governo?.

O Relatório Anual de Gestão (RAG) demanda do gestor a apresentação dos resultados, alcançados ou não, conforme previsto na PAS, e permite redirecionar as ações do PS, quando necessário. A elaboração do RAG é realizada com apoio do Sistema de Apoio à Construção do Relatório de Gestão (SARGSUS), sítio eletrônico no qual os gestores ou técnicos anexam o PS e a PAS, a partir do qual os instrumentos são apreciados pelos conselhos de saúde ${ }^{7,9}$.

Contudo, apesar do reconhecimento da importância dos instrumentos de gestão para o planejamento em saúde, observa-se que há muitos entraves à sua efetivação enquanto base das ações em saúde, destacando-se a pouca compreensão dos conceitos básicos de sua construção e utilização, com consequentes falhas na sua elaboração, dificuldade na integração desses instrumentos entre si, e destes com o Plano Plurianual (PPA) do governo, e fragilidade das práticas de articulação, integração e planejamento ascendente ${ }^{8,11-14}$.

A descontinuidade administrativa e o contexto político de interesses, aliados à baixa capacitação dos gestores para o planejamento, são fatores que também limitam a utilização desses instrumentos como base do planejamento. Além disso, a fraca participação da sociedade, seja no controle social ou como órgão de gestão participativa, agrava ainda mais o distanciamento dos instrumentos de gestão dos seus objetivos ${ }^{10,15}$.

Dada a relevância dos instrumentos de gestão no contexto do planejamento em saúde no SUS, o objetivo desta pesquisa foi analisar a estrutura mínima dos instrumentos de gestão elaborados pelas Secretarias Municipais de Saúde do estado de Santa Catarina. 


\section{Metodologia}

Trata-se de uma pesquisa exploratória de caráter quantitativo, realizada por meio de análise documental. A análise documental consiste em estudar e analisar documentos selecionados e que não sofreram tratamento analítico, com objetivo de compreensão do seu conteúdo, atendo-se às questões de interesse ${ }^{16}$.

Os documentos analisados nesta pesquisa foram o Plano Municipal de Saúde (PMS), que compreende o período de 2014 a 2017; a Programação Anual de Saúde (PAS), de 2017; e o Relatório Anual de Gestão (RAG), referente ao ano de 2017, elaborados pelas 295 Secretarias Municipais de Saúde do estado de Santa Catarina.

Todos os documentos foram obtidos no SARGSUS, sistema eletrônico que auxilia os gestores na construção e publicização dos instrumentos de gestão ${ }^{17}$. O sítio eletrônico do sistema é de domínio público e livre acesso (https://sargsus.saude.gov.br/).

Destaca-se que o PMS de 2014-2017 e a PAS 2017 foram elaborados na gestão municipal do período de 2013 a 2016. Esses instrumentos orientaram o primeiro ano de governo da gestão de 2017 a 2020, sendo desta a responsabilidade de construção do RAG 2017.

A busca do PMS 2014-2017 ocorreu entre 15 e 20 de outubro de 2018, por meio do RAG de
2016, uma vez que o PMS se encontra anexado ao RAG. A busca do RAG de 2017 e da PAS de 2017 foi realizada entre os dias 10 e 20 de junho de 2018. Vale ressaltar que o prazo para envio e entrega dos instrumentos ao SARGSUS vai até 30 de março do ano correspondente ${ }^{7,9}$.

Após a etapa de busca dos instrumentos, procedeu-se a uma leitura integral e criteriosa do conteúdo dos instrumentos encontrados. A análise documental realizada na sequência teve como base a Portaria GM/MS no 2.135/137 e as informações do 'Manual de Planejamento no SUS', a partir das quais foi construído um checklist (quadro 1) com os itens que deveriam constar nesses instrumentos de gestão analisados. Para a construção do checklist do RAG, consideraram-se os itens da estrutura do SARGSUS que o gestor utiliza para sua construção?.

A cada item do checklist foi atribuída uma pontuação igual a 1 (um), caso o item estivesse presente no documento, e igual a 0 (zero) quando não detectado. Foi criada uma planilha no Microsoft Excel, com os municípios nas linhas e os itens avaliados nas colunas. A estrutura mínima de cada instrumento de gestão foi considerada adequada apenas na presença de todos os itens do checklist. Desse modo, era necessário atingir 13 pontos no PMS, 7 pontos na PAS e 13 pontos no RAG.

Quadro 1. Checklist para análise da estrutura mínima dos instrumentos de gestão em saúde

\begin{tabular}{lll}
\hline Plano Municipal de Saúde (PMS) & Programação Anual de Saúde (PAS) & Relatório Anual de Gestão (RAG) \\
\hline I- Período de vigência; & I- Período de vigência & I- Introdução - Considerações Ini- \\
II - Identificação (esfera correspon- & II - Identificação (esfera correspon- & ciais; \\
dente); & dente); & II - Análise e considerações do Ges- \\
III - Ato do Conselho de Saúde que & III - Ato do Conselho de Saúde que & tor sobre Dados Demográficos; \\
avalia o PS; & avalia o Plano de Saúde; & III - Análise e considerações sobre \\
IV - Análise situacional, orientada & IV - Diretrizes, objetivos e indicado- & Morbidade; \\
pelos seguintes temas contidos no & res do PS; & IV - Análise e considerações sobre \\
Mapa da Saúde: & V-Metas do PS anualizadas/revi- & Mortalidade; \\
a) estrutura do sistema de saúde; & sadas; & V-Análise e considerações do Ges- \\
b) redes de atenção à saúde; & VI-Ações que, no ano específico, & tor sobre Prestadores de Serviços ao \\
c) condições sociossanitárias; & garantirão o alcance dos objetivos e & SUS; \\
d) fluxos de acesso; & o cumprimento das metas do Plano & VI-Análise e Considerações dos \\
e) recursos financeiros; & de Saúde; & Profissionais do SUS; \\
\hline
\end{tabular}


Quadro 1. (cont.)

\begin{tabular}{lll}
\hline Plano Municipal de Saúde (PMS) & Programação Anual de Saúde (PAS) & Relatório Anual de Gestão (RAG) \\
\hline f) gestão do trabalho e da educação & VII - Previsão da alocação dos recur- & VII - PAS e Pactuação da Saúde; e \\
na saúde; & sos orçamentários necessários ao & Análise da Execuça Orçamentária; \\
g) ciência, tecnologia, produção e & cumprimento da PAS. & VIII - Análise Sobre a Utilização dos \\
inovação em saúde; & & Recursos; \\
h) gestão; & IX - Análise Sobre os Indicadores \\
V - Definição de diretrizes, objetivos, & Financeiros; \\
metas e indicadores; & X - Análise Sobre Demonstrativo \\
VI - O processo de monitoramento e & Orçamentário; \\
avaliação. & XI - Análise de autoria, quando \\
& houver; \\
& XII - Considerações gerais; \\
& XIII - Recomendações para a próxima \\
& PAS e/ou redirecionamentos para o PS.
\end{tabular}

Fonte: Manual de Planejamento no SUS (2016)9; Sistema de Apoio à Construção do Relatório Anual de Gestão.

As etapas de análise estão ilustradas na figura 1. A análise da estrutura mínima do PMS foi realizada em todos os municípios ( $n=295)$. A busca da PAS e do RAG foi feita apenas para aqueles com PMS considerado adequado. Procedeu-se então à análise da estrutura mínima da PAS $(\mathrm{n}=76)$ e do RAG $(\mathrm{n}=76)$, seguindo o checklist (quadro 1).

Os resultados das análises das estruturas mínimas dos instrumentos de gestão foram sistematizados em tabelas com a frequência simples e absoluta dos itens avaliados.

Figura 1. Etapas da análise dos instrumentos de gestão em saúde

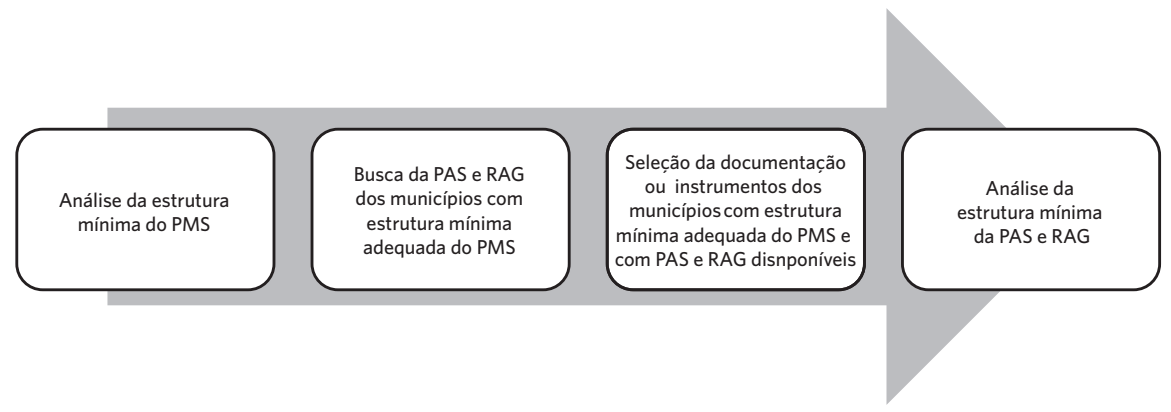

\section{Resultados}

A partir da coleta de dados, foram localizados os três instrumentos de gestão (PMS, PAS e RAG) de 40\% (n=118) dos 295 municípios.
Inicialmente, foi localizado o RAG 2016 de 288 dos 295 municípios catarinenses, a partir do qual se buscou o PMS 2014-2017. A figura 2 ilustra o caminho percorrido nas etapas de busca e análise dos instrumentos de gestão. 
Figura 2. Representação esquemática das etapas de análise dos instrumentos de gestão, com a quantidade de municípios analisados e eliminados da análise



Dos 295 municípios de Santa Catarina, 288 municípios possuíam o RAG 2016, dos quais foram encontrados e analisados 259 PMS, uma vez que 29 municípios não tinham o PMS 20142017 anexado no sistema, ou o PMS verificado era de outro período, impossibilitando sua análise. Os resultados das análises dos itens do checklist dos instrumentos de gestão encontram-se na tabela 1, com frequência absoluta e relativa dos itens encontrados nos instrumentos analisados.

Tabela 1. Frequência absoluta e relativa dos itens encontrados nos instrumentos de gestão avaliados

\begin{tabular}{|c|c|c|}
\hline Item & $\mathbf{n}$ & $\%$ \\
\hline \multicolumn{3}{|l|}{ Plano Municipal de Saúde (N=259) } \\
\hline Ata do Conselho de Saúde que avalia o Plano de Saúde & 207 & 79,9 \\
\hline Análise situacional completa* & 240 & 92,7 \\
\hline Diretrizes, objetivos, metas e indicadores & 175 & 67,6 \\
\hline Monitoramento e avaliação & 185 & 71,4 \\
\hline \multicolumn{3}{|l|}{ Programação Anual de Saúde (N=76) } \\
\hline Ata do Conselho de Saúde que avalia o Plano de Saúde & 64 & 84,2 \\
\hline Diretrizes, objetivos, metas e indicadores do PMS & 58 & 76,3 \\
\hline Metas do PMS anualizadas/revisadas & 71 & 93,4 \\
\hline Definição das ações para alcance dos objetivos e metas do PMS & 73 & 96,0 \\
\hline Previsão da alocação dos recursos orçamentários & 64 & 84,2 \\
\hline \multicolumn{3}{|l|}{ Relatório Anual de Gestão (N=76) } \\
\hline Introdução - considerações iniciais & 72 & 96,0 \\
\hline Análise e considerações sobre dados Demográficos & 71 & 93,4 \\
\hline Análise e considerações sobre Morbidade & 72 & 96,0 \\
\hline
\end{tabular}


Tabela 1. (cont.)

\begin{tabular}{lrr}
\hline Item & $\mathbf{n}$ & $\%$ \\
\hline Análise e considerações sobre Mortalidade & 70 & 92,1 \\
Análise e considerações sobre Prestadores de Serviços & 68 & 89,4 \\
Análise e Considerações dos Profissionais do SUS & 68 & 89,4 \\
PAS e Pactuação da Saúde e Análise da Execução Orçamentária & 29 & 38,1 \\
Análise Sobre a Utilização dos Recursos & 64 & 84,2 \\
Análise Sobre os Indicadores Financeiros & 67 & 84,2 \\
Análise sobre demonstrativo orçamentário & 66 & 86,8 \\
Considerações gerais & 73 & 96,0 \\
Recomendações para a próxima PAS e/ou redirecionamentos para o PMS & 71 & 93,4 \\
\hline${ }^{*}$ Análise situacional completa: apresentou dados dos itens: a) estrutura do sistema de saúde; b) redes de atenção à saúde; c) condições \\
sociossanitárias; d) fluxos de acesso; e) recursos financeiros; f) gestão do trabalho e da educação na saúde; g) ciência, tecnologia, \\
produção e inovação em saúde; h) gestão.
\end{tabular}

As principais inconsistências encontradas na estrutura do PMS foram: anexos do PMS em branco; data de vigência pertencente a outros anos; documentos nomeados como PMS, porém, trata-se do PPA do governo ou da PAS anexada no espaço de anexo do PMS, municípios sem anexos do PMS, instrumentos sem análise situacional e quadros de metas incompletos ou em branco.

Quanto ao RAG e à PAS de 2017, 248 municípios (84\%) tinham o RAG de 2017 disponível no sistema, dos quais, 125 (50,4\%) anexaram o arquivo da PAS 2017 no RAG. Foi analisada a estrutura mínima da PAS e do RAG dos municípios que possuíam a estrutura mínima do PMS 2014 a 2017 e que apresentavam os 3 instrumentos de gestão disponíveis no sistema, correspondendo a 76 municípios. Os resultados dessa análise também se encontram na tabela 1.

A maior parte dos municípios apresentou dificuldades na elaboração das Diretrizes, dos Objetivos, Metas e Indicadores (Domi) do PMS. Consequentemente, considerando sua integração, esse item foi o mais inconsistente na PAS (tabela 1).

Além disso, havia instrumentos sem preenchimento do item 'Previsão da alocação de recursos orçamentários'. Observa-se, nesse sentido, a falta de conhecimento dos gestores e técnicos sobre a interligação entre os instrumentos de gestão do SUS e os instrumentos de governo, visto que a PAS deverá orientar a alocação de recursos para a saúde na LDO e na Lei Orçamentária Anual (LOA) do governo.

Com relação ao preenchimento do RAG 2017, observou-se que 51 municípios não realizaram a totalidade de análises dos itens no relatório (não preencheram os campos de análise dos dados), sendo este composto pelo 'esqueleto' de relatório do próprio SARGSUS, que importa os dados dos demais sistemas de informação do SUS.

O quadro 2 ilustra o preenchimento de alguns RAG no campo final de 'Análise e considerações gerais sobre o Relatório de Gestão', que corresponde ao item 11 do relatório, composto por dois subitens: 11.1 Considerações Gerais; e 11.2 Recomendações para a próxima Programação Anual de Saúde e/ou redirecionamentos para o PS. Nesse item, tendo como base o conjunto de análises realizadas no RAG, o gestor deve apontar possíveis adequações das ações do PMS ou do PAS para o próximo exercício. Entretanto, foram encontradas várias inadequações no seu preenchimento, como 'xxx', '..., 'ok' ou outra frase sem qualquer sentido para esse item, sem quaisquer considerações sobre o relatório apresentado, muito menos recomendações para redirecionamento das ações planejadas. 
Quadro 2. Exemplos de preenchimento do campo 'Recomendações para a próxima Programação Anual de Saúde e/ou redirecionamentos para o Plano de Saúde' do Relatório Anual de Gestão (RAG) de 2017

'xxxx'; '..'; 'ok'

'Nada a comentar'

'Sem recomendações'; 'Nenhuma recomendação'; 'Não há'

'Até o momento não temos Programação Anual de Saúde'

'Elaborar programação anual de saúde'

'PAS 2018'

'O plano está de acordo com o previsto na legislação'

'Que esta análise se aprimorada, revista para o próximo ano'

'Iniciamos os trabalhos do Plano Municipal de Saúde e Programação Anueal de Saúde'

'Seria interessante se cada link, se cada indicador fosse o responsável da informação que pudesse fazer o devido co-

mentário, assim sendo cada profissional teria uma identificação pessoal e o gestor só conferia as colocações e autori-

zava enviar pr conselho'

'Recomenda-se a compatibilização da Programação anual de saúde com o orçamento municipal'

'Algumas adequações precisam ser implementadas e o outras devem ser revistas para o próximo ano'

'Plano Municipal de Saúde e Programação Anual de Saúde são instrumentos importantes para planejamento. Agora com blocos de Custeio e Investimentos facilita o planejar.'

'Os dados epidemiológicos devem estar disponíveis no mesmo momento em que o programa abre para os municípios preencherem'

Nota: Os erros ortográficos são compatíveis com os documentos originais de onde foram retirados.

Apesar de alguns municípios terem informado a necessidade de recomendações para os próximos instrumentos, não foi identificado a que serviços e/ou ações essa necessidade se refere, denotando descompromisso com a qualidade das informações inseridas. Observouse que o preenchimento foi realizado apenas pelo seu caráter obrigatório, impossibilitando a continuidade do processo de planejamento.

O trecho 'Plano Municipal de Saúde e Programação Anual de Saúde são instrumentos importantes para o planejamento. Agora com blocos de Custeio e Investimentos facilita o planejar' reafirma o desconhecimento ou o descaso dos responsáveis pela elaboração dos instrumentos de gestão. Apesar de afirmar a importância do PMS e da PAS, não se faz qualquer citação de redirecionamentos dos instrumentos, conforme solicita o item. Além disso, reduz a importância do planejamento das ações, seu monitoramento e avaliação, declarando que a capacidade de planejar se resume apenas à utilização de recursos dos blocos de financiamento.

A última frase do quadro 2 expressa a dificuldade dos responsáveis na análise e nas considerações dos indicadores do Pacto pela Saúde (item 7 do RAG). No período referido, a interoperabilidade entre o SARGSUS e o Sispacto dificultou a análise dos indicadores pelos técnicos/gestores ${ }^{22}$. Porém, os resultados desses indicadores poderiam ser encontrados no Sistema de Informações do estado de Santa Catarina (http://www.saude.sc.gov.br/index. $\mathrm{php} /$ servicos/gestores-da-saude), reiterando a falta de qualificação adequada para o cargo/ setor de planejamento ou gestão.

Alguns municípios referem não possuir a PAS, instrumento que deveria ser objeto de análise deste relatório (de 2017) e enviado com os devidos redirecionamentos para o conselho de saúde. Deveria, também, orientar a próxima PAS (de 2018) e, posteriormente, a LDO, em abril do referido ano.

Por fim, apenas oito municípios possuíam estrutura mínima adequada nos três instrumentos de gestão analisados, no universo de 295 municípios de Santa Catarina.

\section{Discussão}

O SARGSUS é uma plataforma de apoio aos gestores que, além de auxiliá-los na construção dos instrumentos de gestão, é um importante 
meio de transparência aos cidadãos, sendo seu preenchimento obrigatório. Nesse sítio eletrônico, é possível encontrar o PMS, a PAS e o RAG, além das Resoluções dos Conselhos de Saúde que emitem parecer conclusivo das contas, metas e ações propostas pelo gestor ${ }^{7}$. $\mathrm{O}$ SARGSUS trouxe grandes contribuições para os gestores, facilitando o processo de elaboração do relatório a partir da interoperabilidade com outros sistemas de informação do SUS, trazendo a estrutura mínima requerida na legislação vigente. Com o SARGSUS, o gestor produz e envia os instrumentos para apreciação do Conselho de Saúde por meio eletrônico. Além disso, auxilia no sentido de atender aos prazos legais de sua apresentação junto aos respectivos Conselhos de Saúde e aos demais órgãos de controle externo ${ }^{17}$.

Apesar disso, observou-se neste estudo, além da indisponibilidade de instrumentos no sistema, a presença de muitos problemas em sua estrutura mínima, indicando uma fragilidade do seu desenvolvimento e utilização conforme preconizado na legislação. Chama a atenção o fato de apenas 8 dos 295 municípios catarinenses apresentarem adequação da estrutura mínima requerida nos instrumentos de gestão, uma vez que estes já estão institucionalizados no SUS há mais de dez anos. Esse resultado tem sido observado também em outros estudos realizados no País ${ }^{\mathbf{1 3 , 1 8}}$, que apontam dificuldades por parte dos gestores no desenvolvimento e na aplicação dos instrumentos de gestão, apresentando estrutura fragilizada e utilizando os instrumentos de forma parcial. Apesar dos gestores terem conhecimento do RAG, a sua utilização para o planejamento se demonstra incipiente e normativa ${ }^{13}$.

O fato de $20 \%(n=207)$ dos municípios que continham o PMS não apresentarem a Ata do Conselho Municipal de Saúde (CMS) com a sua aprovação denota a fragilidade do controle social nos municípios. O Conselho de Saúde, como órgão fiscalizador paritário, que atua na formulação de estratégias e no controle e na execução das políticas e de recursos orçamentários, tem poder para contestar e questionar a prestação de contas e das políticas de saúde. Contudo, em muitos casos, seu papel no controle social apresenta-se incipiente, limitando-se apenas como órgão de aprovação dos instrumentos, sem participação nas tomadas de decisões e nos arranjos operacionais do sistema ${ }^{2,19,20}$.

O PMS tem sido referido nas Atas dos conselhos de saúde apenas em sua aprovação e deliberação, sem menção de instrumentalizar o monitoramento e a avaliação das políticas discutidas e aprovadas no PMS ${ }^{19}$. Deste modo, os conselhos de saúde, que são importantes espaços de democracia representativa, acabam se constituindo apenas por instâncias de aprovação de documentos e seguem pautas manejadas por gestores, que muitas vezes são presidentes do $\mathrm{CMS}^{21}$. A Resolução n ${ }^{0} 333 / 03^{22}$ do Conselho Nacional de Saúde dispõe, entre outras questões, sobre a obrigatoriedade da paridade dos conselhos, e, quanto à sua coordenação, coloca que o Presidente deve ser "[...] eleito entre os membros do conselho", não havendo, portanto, impedimento da figura do gestor de coordenar o CMS. Evidencia-se, nesses casos, um conflito de cargos/interesses, pois o próprio gestor fiscaliza e delibera sobre sua própria gestão $0^{23}$.

Corroborando os achados desta pesquisa, outros autores ${ }^{24}$ destacaram que, apesar de a maior parte dos conselheiros relatar pouca dificuldade de analisar documentos, relatórios e orçamentos, cerca de $54,8 \%$ nunca participaram de uma reunião sobre orçamento. Além disso, não se encontra por parte dos conselhos de saúde participação na elaboração e fiscalização dos planos de saúde.

Na construção do RAG 2017 dos municípios de Santa Catarina, 26\% dos presidentes dos CMS eram gestores e $35 \%$ usuários. Em outro estudo ${ }^{25}$, que analisou o controle social por meio do Sistema de Acompanhamento dos Conselhos de Saúde (Siacs), 34\% dos CMS do Brasil são coordenados por gestores, e, ao contrário do que dispõem as normativas, $25 \%$ dos CMS não são paritários, dificultando a fiscalização por parte do controle social. 
Outros estudos $\mathbf{2 6 , 2 7}$ também apontaram fatores que interferem na efetivação do controle social por meio dos conselhos de saúde e destacaram o uso excessivo de linguagem técnica nas reuniões, a restrição de acesso às informações necessárias para a tomada de decisão ou, ainda, desconhecimento dos instrumentos de gestão que regem a política de saúde. As competências dos CMS estão claramente definidas na legislação que normatiza o seu funcionamento, sendo a prestação de contas a principal atividade de controle e avaliação dos conselhos. Porém, a interpretação e o questionamento de um documento como o RAG, por exemplo, exigem um grau de conhecimento técnico nem sempre alcançado pelos conselheiros, resultando em aprovação de documentos com deficiências possivelmente não identificadas. Contribui para essa situação o fato de os instrumentos de gestão chegarem ao CMS já completamente finalizados e com prazos exíguos para aprovação, impedindo sua análise mais minuciosa por parte dos conselheiros, que acabam aprovando-os ‘sob pressão', uma vez que a sua não aprovação pode significar a perda de recursos por parte do município ${ }^{26,27}$.

O Plano Municipal de Saúde consiste no instrumento central de planejamento, que consolida as políticas prioritárias do governo através das suas intenções e dos resultados esperados, embasado por análise situacional ${ }^{4,9}$. No PMS do período de 2014 a 2017, verificou-se que 53\% dos municípios não se adequaram à estrutura mínima disposta em legislação.

A elaboração do PMS se caracteriza por um modelo normativo, ou seja, segue o disposto nos documentos orientadores para sua construção, porém, o instrumento não é utilizado para nortear a gestão ${ }^{\mathbf{2 8}}$. Importante ressaltar que, conforme o parágrafo III do art. $4^{\circ}$, da Lei $n^{0} 8.142 / 90^{\mathbf{1}}$, para que os municípios recebam recursos, é necessário, entre outras coisas, contar com o PS'. É possível inferir, portanto, que a maioria dos munícipios de Santa Catarina elabora o PMS utilizando-se de uma racionalidade normativa, ou seja, apenas para fins de cumprimento da lei e com finalidade de captação de recursos. A racionalidade normativa, em detrimento das racionalidades estratégica e comunicativa, implica a ação em que "os agentes se desobrigam de prestar contas em público de suas intenções e modos de fazer, não atentando para as consequências possíveis dos seus atos"29(20). De tal modo, as pretensões dos sujeitos não são submetidas ao consenso ensejado comunicativamente, representando, segundo Habermas, uma "ética dos fins últimos", e não uma "ética da responsabilidade"29(20).

Resultados semelhantes foram encontrados em outro estudo ${ }^{30}$, que, ao analisar o PMS de 33 municípios mineiros, verificou diversos problemas em sua estrutura, destacando-se a análise situacional com dados defasados em mais de 10 anos, a presença de metas não quantificadas, a ausência de conexão com outros instrumentos de governo, de modo que apenas 3 seguiram os critérios estabelecidos para sua elaboração. Outros autores ${ }^{8}$ encontraram inconsistências entre os problemas elencados na análise situacional, nas diretrizes e nos objetivos do PMS.

Por consequência, a construção do PMS, embasada na racionalidade normativa, com participação social deficiente e sem considerar seu objetivo principal, que é a planificação das políticas em saúde dos municípios, exerce forte influência na elaboração e na compreensão da Programação Anual de Saúde e do Relatório Anual de Gestão por parte dos gestores e dos conselhos de saúde.

De acordo com os resultados do presente estudo, dos 295 municípios, 76 enviaram a PAS e o RAG 2017 para análise e apreciação do CMS no período de coleta, dos quais, apenas oito municípios possuíam estrutura mínima conforme legislação. Entre as inadequações encontradas, verificou-se a existência de instrumentos com data de vigência de anos anteriores, anexos de aprovação do CMS pertencentes a outros documentos e inconsistências na tabela de ações.

A PAS tem por objetivo anualizar as ações do PMS e planificar sobre os recursos financeiros 
que operacionalizam o respectivo plano. Além disso, essa programação orientará a construção da LDO do governo, que tem por objetivo integrar o PPA e a LOA, além de estabelecer as metas e as prioridades da Administração Pública para o próximo ano 9 . O não preenchimento do item 'Previsão da alocação de recursos orçamentários' denota a desconexão da programação com a realidade, a falta de conhecimento dos gestores e técnicos sobre a interligação entre os instrumentos de gestão do SUS e os instrumentos de governo, ou, ainda, o descaso com o planejamento da previsão orçamentária para execução das ações.

Os resultados das ações propostas e execuções orçamentárias da PAS deverão ser expressos e justificados no RAG, que se constitui em importante meio de transparência e controle social e, portanto, não deve ser um fim em si mesmo, não bastando realizar seu preenchimento no sistema para cumprir as obrigações legais, como se tal fosse um fardo. Ele que vai orientar a elaboração da nova PAS, assim como os necessários ajustes no PMS, tornando-se a principal ferramenta para subsidiar o processo de monitoramento e avaliação da gestão do SUS 9,31. Ainda que a análise da adequação da estrutura mínima aqui proposta não tivesse como objetivo a avaliação da qualidade das informações preenchidas pelos municípios, chamou a atenção o descaso com o preenchimento desse documento tão importante do planejamento municipal em saúde, e por isso a necessidade de se destacarem algumas das inconsistências observadas. Além de não apresentarem a análise necessária do que foi (ou não) realizado, os relatórios não orientam qualquer ajuste no PMS e muito menos subsidiam a construção da PAS do ano seguinte, indicando, assim como em outros estudos , $, 18,19^{\text {, }}$ que os instrumentos são preenchidos apenas para a garantia do repasse de recursos, não sendo utilizados no processo de planejamento dos municípios, reforçando a ausência de uma cultura de planejamento.

A desconexão dos instrumentos com a execução das ações, e sua consequente avaliação, também parece evidente em alguns municípios, quando não há 'nada a comentar' nos campos de análise do RAG, ou quando 'não há recomendações' para ajustes do PMS ou para a elaboração da PAS. Mais uma vez, reforça-se que o planejamento nos municípios tem seguido uma lógica mais normativa do que estratégica. A falta de estabilidade no cargo do gestor ou do técnico em planejamento contribui para a desvalorização do planejamento estratégico e dos instrumentos de gestão ${ }^{32,33}$. Em um estudo avaliativo, observou-se que mais da metade dos profissionais que atuavam no planejamento nos municípios catarinenses não possuíam qualificação adequada, sendo, em sua maioria, profissionais não efetivos, resultando na descontinuidade do planejamento, reduzindo sua importância ${ }^{34}$. No cenário municipal, nem sempre há constituição de equipe própria da área de planejamento, o que certamente tem reflexos no processo de planejamento local28.

Por fim, a partir dos resultados obtidos no presente estudo, e corroborando outros $\operatorname{achados}^{35}$, pode-se inferir que o planejamento no nível municipal consolida-se como nó crítico para a efetivação dos princípios da descentralização e da regionalização, além de comprometer a eficiência do sistema de saúde diante dos escassos recursos a ele destinados.

\section{Considerações finais}

A institucionalização dos instrumentos de gestão por meio do PlanejaSUS foi um marco para consolidação da planificação em saúde no Brasil. Porém, seu funcionamento efetivo é diretamente proporcional ao interesse, conhecimento e envolvimento dos gestores e do controle social.

Identificou-se que a maior parte dos municípios catarinenses apresentou falhas importantes na construção dos instrumentos de gestão, apontando, também, fragilidades do planejamento no nível estadual, uma vez que o planejamento deve ser ascendente. 
A despeito da disponibilização de materiais e modelos padronizados pelo MS e pela Secretaria Estadual, com a estrutura mínima dos instrumentos explicitada de forma simples e objetiva, e da padronização da sua construção por meio do SARGSUS, poucos municípios elaboraram seus instrumentos de acordo com essas especificações.

Embora não tenha sido objeto desta pesquisa, verificou-se a pouca participação dos conselhos de saúde no controle, planejamento e fiscalização do governo, denotando a fragilidade do controle social e evidenciando a necessidade de educação permanente aos usuários e profissionais que compõem esses conselhos, para que se apropriem dos conhecimentos necessários para participar de forma mais efetiva nesse processo.

Outro ponto importante a ser destacado é a posição dos gestores enquanto coordenadores dos CMS. Mesmo não havendo proibição legal para que assumam tal posição, não é apropriado que o presidente do conselho fiscalize sua própria gestão, além do jogo de poder implícito na imposição de pautas e deliberações. $O$ fortalecimento do controle social é fundamental para que as instâncias de participação popular previstas no SUS exerçam seu importante papel e qualifiquem o planejamento e o próprio sistema de saúde.

Os instrumentos de gestão em saúde não podem ser apenas documentos a serem burocraticamente preenchidos, sem conexão com a realidade. É necessário institucionalizar a sua construção e seu uso efetivo no planejamento e na execução das ações em saúde, orientando a alocação de recursos orçamentários e identificando as fragilidades e necessidades de ajustes de acordo com a realidade da população.

A realidade apontada com relação ao planejamento nos municípios catarinenses infelizmente não parece ser diferente do que ocorre em outros municípios brasileiros, encontrando eco em resultados de outras pesquisas realizadas em diversos estados do País.

Como limitações desta pesquisa, destacam-se a falta de informações sobre quem elaborou os instrumentos e a forma como estes foram elaborados, o que possibilitaria a diferenciação, por exemplo, entre aqueles construídos de forma participativa e democrática e aqueles provenientes de empresas de consultoria pagas pelo gestor para elaborar os instrumentos para o município. A inadequação com relação à estrutura mínima não necessariamente desqualifica o instrumento como um todo, assim como a sua adequação estrutural não significa que as informações ali preenchidas são compatíveis com a realidade do município e efetivamente subsidiam o planejamento das ações por parte da gestão. Portanto, a análise aqui proposta não permite juízo de valor com relação à qualidade das informações dos instrumentos de gestão.

Apesar disso, o presente estudo contribui para a identificação da necessidade de qualificação do processo de planejamento local, de fortalecimento do controle social por meio dos CMS e de maior controle das ações da gestão municipal por parte dos demais níveis federativos.

\section{Colaboradores}

Fuginami CN (0000-0002-2470-0592)* contribuiu para a concepção e o planejamento do estudo, coleta de dados, análise e a interpretação dos dados, descrição dos dados do artigo. Colussi CF (0000-0002-3395-9125)* contribuiu para a descrição dos dados do artigo, revisão crítica do conteúdo, aprovação final do manuscrito e considerações. Ortiga AMB (0000-0003-1602-1984)* contribuiu para a revisão crítica do conteúdo, aprovação final do manuscrito e considerações.
*Orcid (Open Researcher and Contributor ID). 


\section{Referências}

1. Brasil. Ministério da Saúde. Lei no ${ }^{8} 8080 / 90$. Dispõe sobre as condições para promoção, proteção e recuperação da saúde, a organização e o financiamento dos serviços correspondentes e dá outras providências. Diário Oficial da União. 20 Set 1990. [acesso em 2020 ago 5]. Disponível em: http://www.planalto.gov. br/ccivil_03/leis/18080.htm.

2. Brasil. Ministério da Saúde. Lei ${ }^{\circ} 8.142 / 90$. Dispõe sobre a participação da comunidade na gestão do Sistema Único de Saúde (SUS) e sobre as transferências intergovernamentais de recursos financeiros na área da saúde e dá outras providências. Diário Oficial da União. 31 Dez 1990. [acesso em 2020 ago 5]. Disponível em: http://www.planalto.gov.br/ccivil_03/leis/ L8142.htm.

3. Brasil. Ministério da Saúde. Portaria n ${ }^{\circ} 399$, de 22 de fevereiro de 2006: Divulga o Pacto pela Saúde 2006 - consolidação do SUS e aprova as Diretrizes Operacionais do Referido Pacto. Diário Oficial da União. 23 Fev 2006. [acesso em 2020 ago 5]. Disponível em: https://bvsms.saude.gov.br/bvs/saudelegis/gm/2006/ prt0399_22_02_2006.html.

4. Brasil. Ministério da Saúde. Sistema de planejamento do SUS: uma construção coletiva: orientações gerais para a elaboração de instrumentos de planejamento. Brasília, DF: Ministério da Saúde; 2009.

5. Trevisan L, Junqueira LAP. Construindo o "pacto de gestão" no SUS: da descentralização tutelada à gestão em rede. Ciênc. Saúde Colet. 2007; 12(4):893-902.

6. Vicentine FB, Delatorre T, Feltrin AFS, et al. Aspectos da gestão que influenciam o processo de planejamento municipal e regional do Sistema Único de Saúde. Medicina (Online) 2018; 51(1):1-11. [acesso em 2020 set 27]. Disponível em: http://www.revistas.usp. br/rmrp/article/view/150073.

7. Brasil. Ministério da Saúde. Portaria no 2.135 , de 25 de setembro de 2013. Estabelece diretrizes para o processo de planejamento no âmbito do Sistema Único de Saúde (SUS). Diário Oficial da União. 26
Set 2013. [acesso em 2020 ago 5]. Disponível em: http://bvsms.saude.gov.br/bvs/saudelegis/gm/2013/ prt2135_25_09_2013.html.

8. Galindo AC, Gurgel AM. Planejamento de ações de saúde do trabalhador no SUS: uma análise dos planos municipais de saúde da VI Regional de Saúde de Pernambuco. RECIIS (Online) 2016; 10(4):1-20. [acesso em 2019 jun 22]. Disponível em: https://www.reciis. icict.fiocruz.br/index.php/reciis/article/view/1155/ pdf1155.

9. Brasil. Ministério da Saúde. Manual de planejamento no SUS. Brasília, DF: Ministério da Saúde; 2016.

10. Araujo RF, Costa JMBS, Cruz MM, et al. Avaliabilidade da compatibilização de indicadores e metas dos instrumentos de planejamento do Ministério da Saúde. Saúde debate. 2018; 42(118):566-78.

11. Anunciação FC, Souza MKB. Planejamento em saúde: Percepções e entendimentos sobre o plano municipal de saúde. Rev. Baiana Saúde Pública 2011; 35(4):845858.

12. Freitas GM, Baratieri T, Pilger C. Planos de Saúde dos municípios de uma regional de saúde do Paraná. Rev. Enferm. UFSM 2013; 3(1):122-132.

13. Pinafo EG, Carvalho B, Nunes EFPA, et al. Gestor do SUS em município de pequeno porte no estado do $\mathrm{Pa}-$ raná: perfil, funções e conhecimento sobre os instrumentos de gestão. Espaço para Saúde. Rev. Saúde Pública Paraná 2016; 17(1):130-137.

14. Paulo LFA. Planejamento estratégico e gestão por resultados: o caso do Ministério da Saúde. Physis 2016; 26(3): 981-1007.

15. Ricardi LM, Shimizu HE, Santos LMP. As Conferências Nacionais de Saúde e o processo de planejamento do Ministério da Saúde. Saúde debate 2017; 41(3):155-170

16. Kripka RML, Scheller M, Bonotto DL. Pesquisa do- 
cumental na pesquisa qualitativa: conceitos e caracterização. Rev. Investigaciones UNAD Bogotá 2015; 14(2):55-73.

17. Brasil. Ministério da Saúde. Portaria n ${ }^{\circ} 575$, de 29 de março de 2012: Institui e regulamenta o uso do Sistema de Apoio ao Relatório Anual de Gestão (SARGSUS), no âmbito do Sistema Único de Saúde (SUS). Diário Oficial da União. 30 Mar 2012.

18. Almeida ARC, Souza MKB. Processo de construção e análise dos relatórios anuais de gestão: dificuldades, avanços e desafios. Rev. Baiana Saúde Pública 2013; 37(4):852-868.

19. Kleba ME, Comerlatto D, Frozza KM. Instrumentos e mecanismos de gestão: contribuições ao processo decisório em conselhos de políticas públicas. Rev. Adm. Pública 2015; 49(4):1059-1079.

20. Araujo MAD. Responsabilização pelo controle de resultados no Sistema Único de Saúde no Brasil. Rev. Panam. Salud Public. 2010; 27(3):230-6.

21. Finkler RU, Ribeiro ER. Controle social da saúde no Brasil. Cad. Saúde e Desenvolvimento 2014;3(2):6277.

22. Conselho Nacional de Saúde. Resolução do Conselho Nacional da Saúde (CNS) n $333 / 2003$. Aprova as diretrizes para criação, reformulação, estruturação e funcionamento dos Conselhos de Saúde. Diário Oficial da União. 4 Dez 2003. [acesso em 2020 ago 5]. Disponível em: http://bvsms.saude.gov.br/bvs/saudelegis/cns/2003/res0333_04_11_2003.html.

23. Tribunal de Contas do Estado de São Paulo. Guia de orientação aos membros do conselho municipal de saúde. São Paulo [internet]. 2019 [acesso em 2019 jul 3]. Disponível em: https://www.tce.sp.gov.br/sites/ default/files/publicacoes/Guia\%20de\%20orient\%20 Cons\%20Munic\%20Saude\%202020.pdf.

24. Saliba NA, Moimaz SAS, Ferreira NF, et al. Conselhos de saúde: conhecimento sobre as ações de saúde. Rev. Adm. Pública. 2009; 43(6):1369-1378.
25. Silva Junior ER. Controle social na saúde pública: a relação entre as características dos conselhos de saúde e o resultado da análise do relatório anual de gestão [TCC]. Brasília, DF: Escola Nacional de Administração Pública; 2016.

26. Santos CL, Santos PM, Pessali, HF, et al. Os conselhos de saúde e a publicização dos instrumentos de gestão do SUS: uma análise dos portais das capitais Brasileiras. Ciênc. Saúde Colet. [internet]. 2019 [acesso em 2019 jun 22]. Disponível em: http://www. cienciaesaudecoletiva.com.br/artigos/os-conselhos-de-saude-e-a-publicizacao-dos-instrumentos-de-gestao-do-sus-uma-analise-dos-portais-das-capitais-brasileiras/17211?id=17211.

27. Pinheiro R. A Dinâmica dos Conselhos Municipais de Saúde do Estado do Rio de Janeiro: três estudos de caso: Angra dos Reis, Resende e Bom Jesus do Itabapoana. Rev. Adm. Pública 1966; 30(5):62-100.

28. Carvalho EMR. Usos e "desusos" do planejamento: uma análise sobre planos municipais de saúde [dissertação]. Salvador: Universidade Federal da Bahia; 2014.

29. Riviera FJU, organizador. Agir comunicativo e planejamento social: uma crítica ao enfoque estratégico. Rio de Janeiro: Fiocruz; 1995.

30. Cruz CSS, Dupim SLM, Nascimento ALD, et al. Análise dos Planos Municipais de Saúde dos 33 municípios sob a jurisdição da Superintendência Regional de Saúde de Diamantina no período de 2014 a 2017. Rev. Vozes Vales. 2017; 12:1-14.

31. Garcia PT, Reis RS. Gestão pública em saúde: o plano de saúde como ferramenta de gestão. São Luís: Edufma; 2016.

32. Fenili R, Correa CEG, Barbosa L. Planejamento Estratégico em saúde: ferramenta de gestão para o complexo de regulação em saúde. Rev. Eletrônica \& Saúde 2017; 8(1):18-36.

33. Barbosa NCT, Cordeiro BC, Abrahão AL, et al. Educação em saúde: o uso da matriz swot para análise de 
projetos. Rev. Enferm. UFPE [internet]. 2017 [acesso em 2019 ago 7]; 11(11):4298-304. Disponível em: https://periodicos.ufpe.br/revistas/revistaenfermagem/article/view/25148-50449-1-SM.doc/24625.

34. Berreta IQ, Lacerda JT, Calvo MCM. Modelo de avaliação da gestão municipal para o planejamento em saúde. Cad. Saúde Pública 2013; 27(11):2143-2154.
35. Pinafo E, Carvalho BG, Nunes EFPA. Descentralização da gestão: caminho percorrido, nós críticos e perspectivas. Ciênc. Saúde Colet. 2016; 21(5):1511-1524.

Recebido em 21/01/2020

Aprovado em 07/06/2020

Conflito de interesses: inexistente

Suporte financeiro: não houve 